\title{
Using $\mathrm{min} / \mathrm{max}$ autocorrelation factors of survey-based indicators to follow the evolution of fish stocks in time
}

\author{
Mathieu Woillez ${ }^{1, a}$, Jacques Rivoirard ${ }^{2}$ and Pierre Petitgas ${ }^{1}$ \\ 1 IFREMER, Département EMH, BP 21105, 44311 Nantes Cedex 03, France \\ 2 Mines-ParisTech, Centre de Géosciences/Géostatistique, 35 rue St Honoré, 77300 Fontainebleau, France
}

Received 15 July 2008; Accepted 22 January 2009

\begin{abstract}
Fisheries research monitoring surveys provide an ensemble of measurements on fish stocks and their environment. Because the interannual variability in such survey-based indicators is high and because diagnostics on fish stocks cannot be based on noise, our concern is to make use of what is continuous in time to obtain a reliable diagnostic. In this paper, we show how min/max autocorrelation factors (MAFs) can be useful for assessing the status of a fish stock. Indeed, MAFs will allow us to (i) summarize the multivariate indicator signals into orthogonal factors that are continuous in time, (ii) select those indicators that carry the major signal in time, and (iii) forecast stock status by modelling the time continuity of the MAFs. These different potential uses of MAFs in an indicator-based approach to assessment were illustrated with North Sea cod, for which a suite of biological and spatial indicators are available over a 21-year survey series.
\end{abstract}

Key words: Min/max autocorrelation factors / Time correlation / Survey-based indicators / Fish stock diagnostics

\section{Introduction}

The indicator-based approach to fish stock assessment uses many indicators that characterize different attributes of a fish stock in order to assess its status (Jennings 2005). The assessment is then a multivariate time series problem, that requires adequate methods to analyse the trends or changes occurring over time. In this context we show the potential for using the $\min / \max$ autocorrelation factors (MAFs). We focus on the use of indicators derived from research surveys.

MAFs are a multivariate statistical method, first developed to separate signals from noise in multivariate imagery observations (Switzer and Green 1984). Shapiro and Switzer (1989) adapted this method to analyse and extract trends from multiple times series. When applied to a time series, $\min / \max$ autocorrelation factors analysis (MAFA) decomposes the set of initial variables into a series of factors (the MAFs), in which autocorrelation decreases from the first factor to the last. Hence the very first factors extract the part of the variables that is the most continuous in time. MAFA amounts to applying a double principal components analysis that exploits the order of the time series and therefore the autocorrelation (Shapiro and Switzer 1989; Conradsen et al. 1985).

MAFA has already been used for a wide variety of applications. In ecology, MAFA has been used to detect change in the composition of a multi-species community (Solow 1994) or

\footnotetext{
a Corresponding author: mathieu.woillez@ifremer.fr
}

coincident changes in different components of the ecosystem (Pearce and Frid 1999). In fisheries, Erzini (2005) explained fishery catches using multiple time series of environmental parameters. He also compared MAFA with Dynamic Factor Analysis (DFA), which is a multivariate time series method that can deal with short non-stationary series (Zuur et al. 2003a; Zuur et al. 2003b; Zuur and Pierce 2004). Both techniques, available in the Brodgar software package (http://www.brodgar.com/), provided coherent results (Erzini 2005; Erzini et al. 2005). In acoustics, MAFs were put to a different use (Bouleau 2005). Layers of acoustic data were combined into MAFs in order to investigate correlations with trawl haul catches. Other applications of MAFs can also be found in the geosciences (e.g., Desbarats and Dimitrakopoulos 2000; Desbarats 2001).

The previous applications used MAF to combine original variables and to construct continuous time series of multivariate factors. Here, in addition to combining indicators, we develop two more useful purposes for MAF in the indicatorbased assessment context. These are (i) to select those indicators that carry the major signals out of the suite of indicators available, and (ii) to forecast the future status of the stock based on kriging the MAFs. Löfgren et al. (1993) proposed forecasting MAFs based on ordinary least squares. Here we suggest kriging after having modelled the variogram of the MAFs. The three uses of MAFs are applied to North Sea cod stock data to demonstrate their utility for assessing the stock using a suite of biological and spatial indicators. The indicators 
were derived from the 21-year (1985-2005) series of International Bottom Trawl Surveys over the North Sea.

\section{Method description}

\subsection{Derivation of MAFs}

Consider multiple time series each corresponding to a particular variable (a survey-based indicator of a fish stock attribute). The surveys are annual, so the time step of the series is a year. The MAFs are particular linear combinations of these variables that have the properties of remaining uncorrelated with each other at all times (as would be the principal components in a PCA) and also of being uncorrelated with each other for a chosen time lag (Shapiro and Switzer 1989). We shall consider this time lag to be a year, the time step of the survey series. Consequently, the cross-correlation between MAFs at other lags is strongly reduced, although its absence is not guaranteed. Moreover, the MAFs are computed so that the first MAF (MAF1) has the highest autocorrelation at the time lag (smallest variogram value), the second MAF (MAF2) has the second highest autocorrelation while being uncorrelated with MAF1, etc.

MAFs can be obtained by applying two PCAs: one to transform the initial variables into principal components (PCs), the other to obtain the MAFs from the increments of the PCs at the chosen time lag. This second step maximizes/minimizes the variance of the increments of the PCs and therefore their autocorrelation. Each MAF is normalized to a variance of 1 . Like a PC, a MAF is equivalent to its opposite (the MAF with changed sign can be obtained by changing the sign of each coefficient of its linear combination) because the variogram at the computation lag remains unchanged. A MAF that was monotonic over a time series could appear to be either increasing or decreasing. Similarly, a MAF with an extreme in the middle of a time series may present either a maximum or a minimum.

\subsection{Robust MAFs}

The number of MAFs cannot exceed the number of variables or the number of year increments (no. years -1 ). If the number of variables is larger than the number of years, the time series of MAF $k(k=1,2, \ldots)$ will have a period equal to (no. years -1$) \times 2 / k$, which may be biologically meaningless.

To prevent such overfitting to the very detailed values of the variables and to increase the significance of the MAFs, we computed them while adding a repeated random white noise to the variables. The white noise was added to the variables after normalization. The white noise was normally distributed with a zero mean and a variance equal per default to $0.1 \times$ (no. indicators / (no. years -1$)$ ) vanishing for a long series. The MAFs were calculated for a given number of realizations (1000) with independent white noises. The final MAF $k$ was obtained by averaging the MAFs over rank $k$ over all realizations as follows: 1) the MAFs of rank $k$ were made consistent over all realizations by giving them the same sign (to avoid the tendency of some MAFs to increase in time, while others decrease); 2) the coefficients for each contributing variable were averaged to their median value (more robust than their mean value); 3 ) the resulting median MAFs $k$ were normalized to a variance of 1 . The final MAFs were a median profile of the MAFs over all realizations.

\subsection{Selection of informative variables}

The MAFs were also used to select the most continuous original variables, which contributed to the first MAFs. The variables with highest continuity were selected based on their loadings on the first $p$ MAFs and the one-lag variograms of these. The continuity of variable $j$ in time was estimated by:

$$
c_{j}=\sum_{k=1}^{p} \lambda_{k, j}^{2}\left(1-\mu_{k}\right),
$$

where $\lambda_{k, j}$ denotes the loading of variable $j$ on MAF $k$ and $\mu_{k}$ the one-lag variogram value of MAF $k$. The continuity index $c_{j}$ is based on different structural components in the time series as different MAFs are used. The loadings are used for interpreting the MAFs. The $c_{j}$ are used to select the original variables that are the most representative of the changes occurring along the time series. Indicators were ranked in descending order of their continuity index $c_{j}$, so some could then be chosen. The selection of a few indicators among the multiple set of indicators made it possible to extract the drivers of the stock history.

\subsection{Forecasting}

MAFs were also used for forecasting the status of the fish stock. Each MAF was forecasted for 6 years ahead using ordinary kriging with a unique neighbourhood, after modelling its variogram. Assuming that the distribution of the indicators and thus that of the MAFs was Gaussian, the $95 \%$ confidence interval of the forecasted MAF was +/- 2 kriging standard deviation.

For the one-year-ahead forecast $n$, the MAF $k$ was calculated in two ways using the years $\leqslant n-1$. On one hand, it was estimated by kriging (forecasted value). On the other hand, the value of the MAF was determined by using the indicator values for the year $n$ and the loadings determined from the years $\leqslant n-1$ (observed value). When the difference between the forecasted and the observed values was high relative to the kriging standard deviation, the year $n$ was considered to be inadequately described by the model, which was indicative of a change in the time series.

\section{Example applications to North Sea cod}

MAFA was applied to the North Sea cod stock, for which indicators of abundance, length, maturity, mortality and spatial distribution were estimated using the survey data of the North Sea International Bottom Trawl Surveys (IBTS) coordinated by the International Council for the Exploration of the 


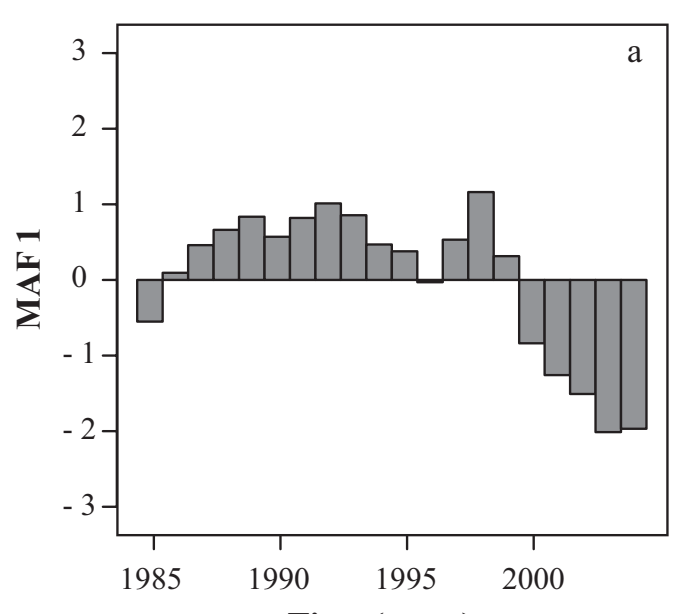

Time (years)

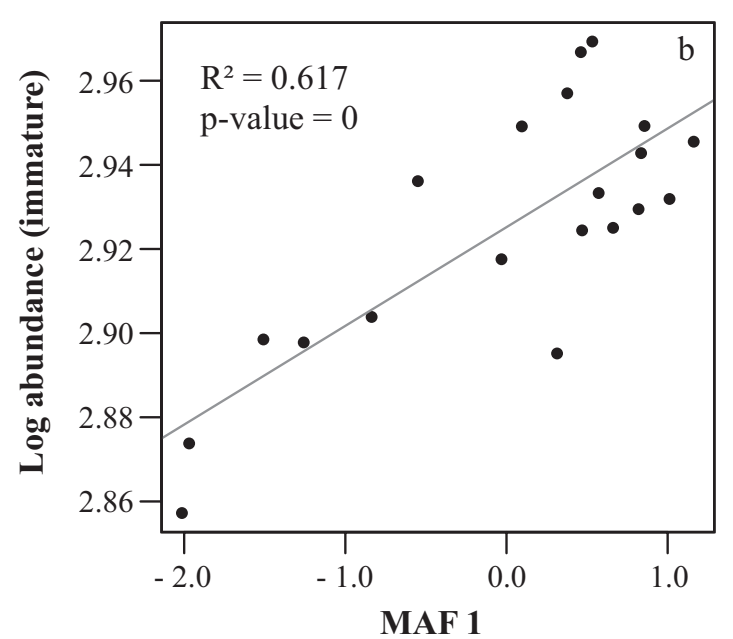

MAF 1

Fig. 1. (a) MAF 1 time series built from spatial indicators of immature North Sea cod over the period 1985-2004 and (b) significant linear regression adjusted between the logarithm of the corresponding abundance and MAF1.

Table 1. Maturity at age used to determine the functional groups of immature and mature fish in the North Sea cod stock.

\begin{tabular}{ccccccc}
\hline Age & 1 & 2 & 3 & 4 & 5 & 6 \\
\hline $\begin{array}{l}\text { Proportion of } \\
\text { mature fish }\end{array}$ & 0.01 & 0.05 & 0.23 & 0.62 & 0.86 & 1.00 \\
\hline
\end{tabular}

Sea (ICES IBTS quarter 1 data). This survey series spanned 21 years, 1985-2005. We considered 9 spatial indicators to characterize location, dispersion, aggregation in the spatial distributions (Woillez et al. 2007a; Woillez et al. 2009). These were computed for the functional group recruits (age 2, as the survey gear has a low selectivity for age 1), immature and mature fish (Table 1). We also considered 8 biological indicators (Cotter et al. 2009). The 3 indicators of abundance were the logarithms of the numbers of immature and mature fish and of recruits. The 3 length indicators were the average length in the population and the first and third quartile of fish length (Lbar, L25, L75). The indicator of maturity was the length at which 50 percent of the fish were mature (L50 at maturity). The indicator of mortality $(Z)$ was the annual apparent mortality estimated by the log ratio of the summed abundance for ages 1-5 over the summed abundance for ages 2-6. Example applications of MAFs were performed on the multiple indicator time series to show how a number of questions could be answered: how can all the indicators be summarized to construct the stock history, what were the major changes and what indicators were involved, and can the future state of the stock be predicted based on the past history?

\subsection{Combining raw indicators into time continuous multivariate indicators}

We considered the 9 spatial indicators for the functional group composed of the immature fish. MAFs were calculated for the period 1985-2004 to summarize the multiple time series, detect changes in the spatial organisation and identify which indicators were responsible for the detected change. The
Table 2. MAF1 loadings of spatial indicators computed from survey data on immature North Sea cod stock over the period 1985-2004.

\begin{tabular}{lc}
\hline Spatial indicators & Loadings \\
for immature cod stock & for MAF1 \\
\hline Positive area & 1.023 \\
Inertia & -0.325 \\
Anisotropy & 0.376 \\
Longitude of the centre of gravity & 0.489 \\
Latitude of the centre of gravity & -0.069 \\
Microstructure & -0.078 \\
Equivalent area & 0.620 \\
Spreading area & -0.140 \\
Number of patches & 0.195 \\
\hline
\end{tabular}

calculation of MAFs used the robust MAF estimation procedure (white noise added and 1000 realizations). MAF1 was the most continuous factor with a one-lag variogram value of 0.10 , while that for MAF2 was 0.40. MAF1 showed a marked change at the end of its series, since the year 2000, indicating a change in the spatial distribution (Fig. 1a). The indicators that contributed the most to MAF1 (higher loading values, Table 2) and which were responsible for the change were: positive area, equivalent area and longitude of the centre of gravity. In addition, MAF1 was significantly correlated with the abundance of the immature fish (Fig. 1b), indicating that the changes detected in the spatial distribution were also reflected in the abundance.

\subsection{Selection of informative indicators}

We considered all 35 available indicator time series (period 1985-2004) for the entire population: the 9 spatial indicators for the recruits, immature and mature groups and the 8 biological indicators. MAFs were used to select a few indicators among the long list that showed a major signal of change in the population. This was done in three steps: 1) an initial selection of the indicators; 2) the calculation of MAFs on this restricted list; and 3) the calculation of the continuity index $c_{j}$ (Eq. (1)). 


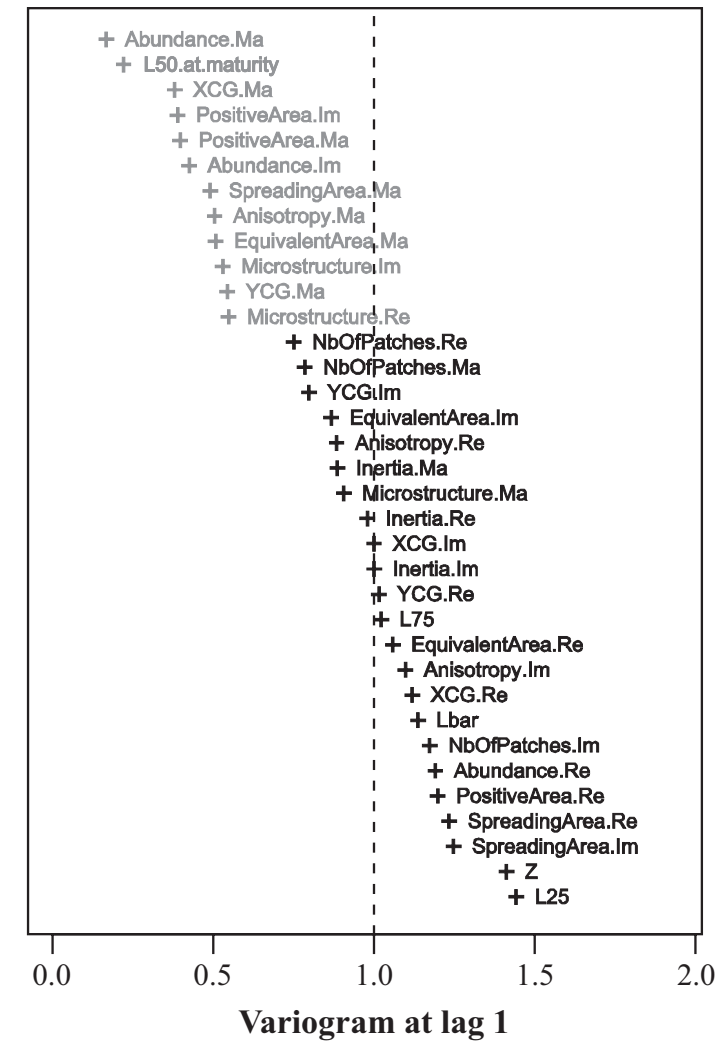

Fig. 2. Plot representing the indicators describing the North Sea cod stock, ordered according to their time correlation for the period 19852004. In grey, the most continuous indicators in time, which were retained in the first selection step.

The one-lag variogram for each indicator was computed, scaled to the indicator variance and ranked (Fig. 2). The ranked indicators were grouped. The 12 indicators in the group with highest continuity at lag-one were selected (these had variogram values in the range $0.167-0.547)$. MAFs were then calculated over the period 1985-2004 using the 12 selected indicators. The calculation used the robust MAF construction procedure (white noise added and 1000 realizations). The first two MAFs had low one-lag variogram values, 0.03 and 0.18 respectively. The continuity index $c_{j}$ was calculated for each of the 12 indicators on the first 2 MAFs and indicators were ranked by ascending order of $c_{j}$ (Fig. 3). Note that the ranking is not the same as that of the loadings because the MAF variogram values also play a role in determining $c_{j}$. The first 5 indicators were selected to represent the history of the stock (Fig. 4). A long-term trend is clear for all indicators, meaning that the stock has been experiencing continuous change. The indicator L50 at maturity showed a decreasing continuous trend over the whole of the 1985-2004 period, while the latitude of centre of gravity of mature fish shifted towards the North. The two particular values of the latitude of the centre of gravity at the beginning of the series can not be considered as reliable because these years present cod density distributions that are more skewed (due to the presence of outliers) than those of the other years. The other indicators (logarithm of the abundance of mature, microstructure of immature and anisotropy of mature fish) showed a rapid decline

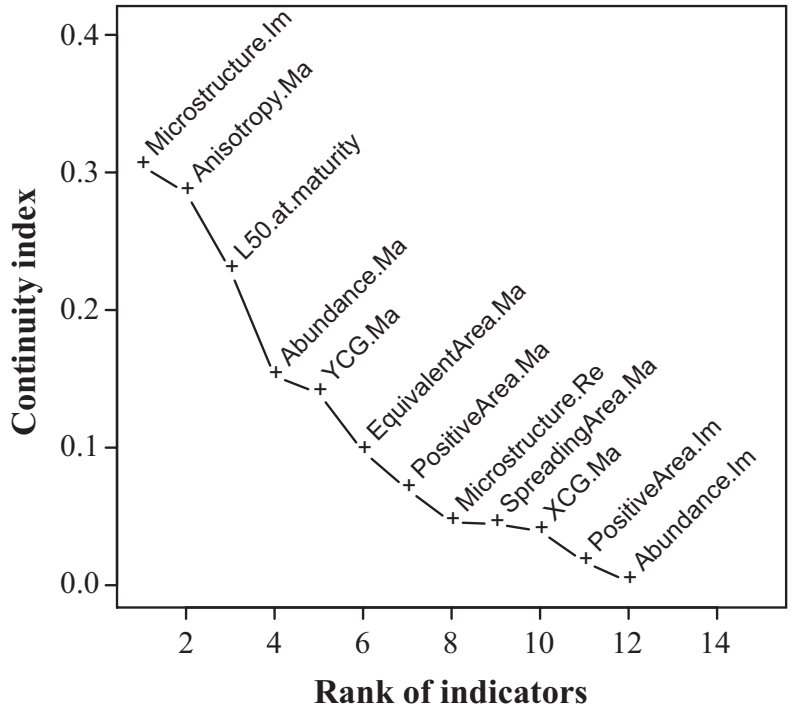

Fig. 3. Indicators ranked according to their continuity index on the first two MAFs.

until the mid-90s. The logarithm of the abundance of mature fish showed another decline after 2000, while the other two spatial indicators stabilized at around their mid-90s value. This could suggest that the different attributes of a stock do not necessarily change on the same time scale.

\subsection{Forecasting}

We considered the 2 first MAFs constructed in the previous section and forecasted their values by kriging. For each MAF, a variogram was estimated and modelled (Fig. 5). Note that, since each MAF has a variance of 1 , small values of the variogram at short time lags are compensated by large values at larger time lags. This explains the large MAF1 variogram values at large time lags. The MAF values for the next 6 years (2005-2010) were estimated by kriging using the variogram model and all historical MAF values (1985-2004). For the forecasted years, the kriging error and confidence interval increased with the time separation from the historical values. The forecast for MAF1 followed a continuation of the change observed in the MAF since 2000. MAF1 was explained by the logarithm of the abundance of mature cod, L50 at maturity and longitude of the centre of gravity (loadings in Table 3 ). Therefore, the prediction was that the logarithm of the abundance of mature fish and the L50 at maturity were expected to decrease even more and the centre of gravity to move still further to the East (positive loading on MAF1). The forecast for MAF2 was the mean of the MAF historical series. Although it is doubtful that the long-term 6-year forecast would be realistic, the short-term forecast (for the first years just after the end of the series) is worthwhile. The first forecasted year (2005) was of particular interest. The 2005 forecasted values for MAFs 1 and 2 continued the trend observed in the MAFs of previous years. Therefore, no change was expected in 2005 compared with previous years. This prediction was validated using the observed 2005 MAF values (Fig. 5). As MAFs are a linear combination of the original indicators, once the coefficients of 

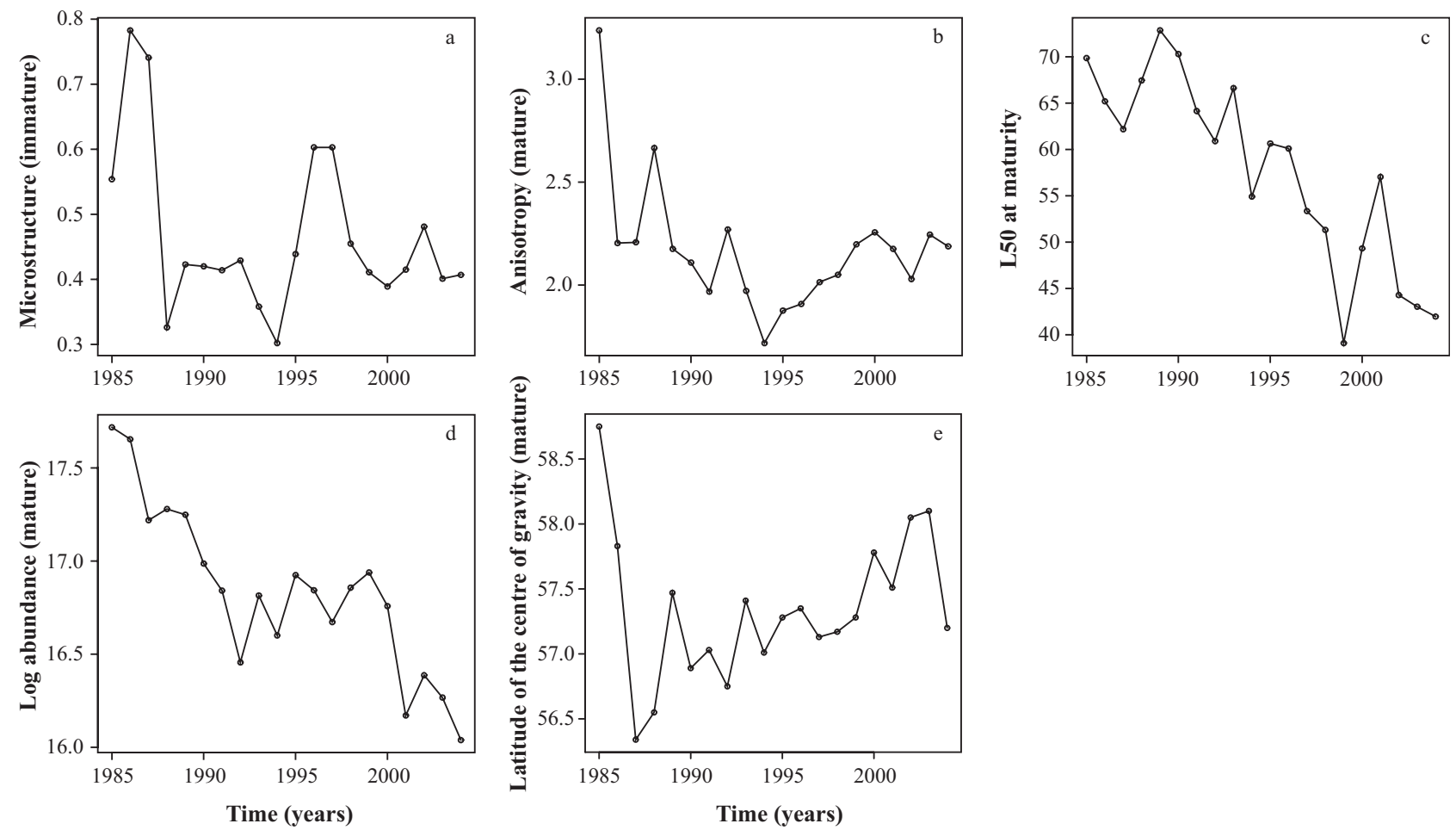

Fig. 4. Time series of selected indicators with the highest continuity on the MAFs: microstructure of the immature fish (a), anisotropy of the mature fish (b), L50 at maturity (c), logarithm of the abundance of the mature fish (d) and lattitude of the centre of gravity of the mature fish (e).

Table 3. Loadings on MAFs $1 \& 2$ for the selected indicators representing the North Sea cod stock over the period 1985-2004.

\begin{tabular}{lrr}
\hline \multicolumn{1}{c}{ Indicators } & \multicolumn{2}{c}{ Loadings } \\
\cline { 2 - 3 } \multicolumn{1}{c}{ for the cod stock } & MAF1 & MAF2 \\
\hline Microstructure (immature) & 0.040 & -0.624 \\
Anisotropy (mature) & -0.018 & -0.605 \\
Length at 50\% maturity & -0.363 & 0.391 \\
Log abundance (mature) & -0.419 & -0.087 \\
Latitude of the centre of gravity (mature) & 0.215 & -0.033 \\
Equivalent area (mature) & -0.001 & -0.353 \\
Positive area (mature) & -0.020 & 0.298 \\
Microstructure (recruit) & -0.128 & -0.203 \\
Spreading area (mature) & 0.192 & 0.133 \\
Longitude of the centre of gravity (mature) & 0.382 & -0.151 \\
Positive area (immature) & 0.017 & 0.146 \\
Log abundance (immature) & -0.053 & -0.017 \\
\hline
\end{tabular}

each indicator of the MAF are determined (here from the period 1985-2004), the MAF value can be estimated for a new year using these coefficients and the new values for the indicators for that year. The observed MAF for 2005 was calculated in this way and compared well with the kriging forecast.

\section{Software}

\subsection{Description}

Three scripts in $\mathrm{R}$ language ( $\mathrm{R}$ development Core Team 2005) are attached as an on-line supplement to this paper. The scripts will allow the computations explained in the present study to be reproduced and can help to implement a MAF application.

- MAF_combine.r allows a combined indicator to be built using MAFs on a suite of indicators, e.g. the spatial indicators.

- MAF_select.r is designed to use the MAFs to select a set of indicators within a large list of indicators.

- MAF_forecast.r allows MAFs to be used in a forecasting procedure to assist in diagnostics on a fish stock.

The above scripts use functions stored in a separate file, MAF_functions.r, that must be loaded into the user's $R$ workspace as is done for the scripts. One of these functions computes the MAFs from a double call of the standard R function for PCA (prcomp).

\subsection{Inputs}

Input data should have the specific format of the EU project FISBOAT. The script MAF_combine.r works with tables of indicators organised by age and year (these names must be present in the header). The scripts MAF_select.r and MAF_forecast.r need tables of indicators that are built by year only (specified column name). Age or functional group must be incorporated into the indicator column name.

\subsection{Outputs}

MAF_combine.r produces results by age or functional group; these comprise the retained MAF time series, their 

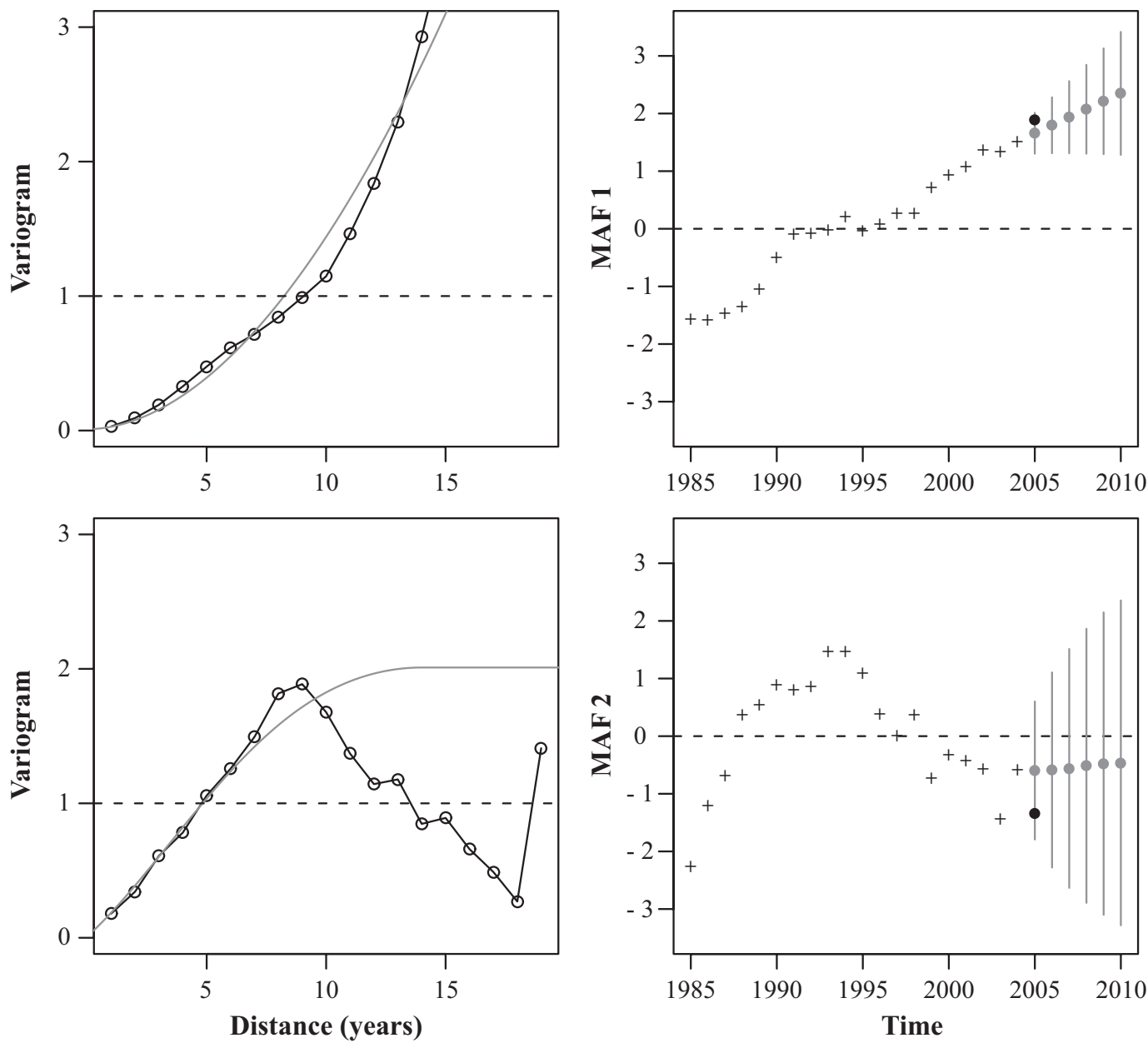

Fig. 5. MAF forecast for North Sea cod using kriging. Left: experimental variograms of MAFs 1 and 2 and fitted models. The model for MAF1 is: nugget $(0.01)+0.018$ year $^{\wedge} 1.9$; that for MAF 2 is: nugget $(0.01)+$ spherical (range $=14$, sill $\left.=0.5\right)$. Right: MAF time series (black crosses) with kriging forecasts (grey points). The grey vertical lines represent the $95 \%$ confidence interval defined by $+/$ - twice the kriging standard deviation. For the forecasted year, 2005, the black point is the observed value for the year using the loadings defined from the previous years.

corresponding variograms and the loadings of each contributing indicator. Regression analysis is then performed between the MAFs and the logarithm of the abundance.

MAF_select.r first proceeds by ranking the indicators according to their one-lag variogram value. The user sets the number of indicators to be retained. The retained indicators are then combined into MAFs. The one-lag variogram value of the retained MAFs and the loadings of each indicator are saved, the continuity index is computed and the indicators are ranked in descending order of the continuity index. The time series of the indicators that present the highest continuity are plotted.

MAF_forecast.r is similar to MAF_select.r until the lines of MAF computation. Then, for each MAF considered, the variogram of the MAF is modelled. Model parameters have to be provided through an interactive dialogue in $\mathrm{R}$. The forecast is performed and the resulting time series is plotted, as are the MAF variogram and its fitted model. The one-year-ahead forecast value, the upper and the lower confidence interval values and the observed value for this year (here 2005) are saved in an R object for each of the MAFs considered.

\subsection{Controls and options}

For all scripts, the indicators to be used in the data input table are selected by their name at the beginning of the script. The number of realizations (default 1000), the proportion giving the amount of variance for the white noise (default $0.1 \times($ no. indicators $/($ no. years -1$)))$ and the number of MAFs to retain (default 2) can be modified. For the scripts MAF_select.r and MAF_forecast.r, the first step for selecting indicators keeps the most continuous indicators based on their one-lag variogram value. The user decides how many indicators he wants to keep based on the ranked indicator one-lag variogram values. For the script MAF_select.r, the user decides how many MAFs are to be used to compute the indicator continuity index. For the script MAF_forecast.r, the user provides the parameters to be used for modelling the variograms.

\section{Practical guidelines}

This method is direct and automatic. However, it is sensitive to the proportion giving the amount of variance for the 
white noise and also slightly sensitive to the randomisation (the seed for the generation of random values is provided to allow users to reproduce the realizations exactly). The main interest of this method is to reduce a set of indicators by extracting the most time-continuous MAFs. Its weakness is in the number of indicators relative to the size of the time series, since the white noise that needs to be added for the robust construction of MAFs has to increase with the number of indicators.

\section{Discussion - Conclusion}

In the context of an indicator approach, $\min / \mathrm{max}$ autocorrelation factors appear to be a useful method for seeking and making use of the continuity of indicator time series. Compared to a finer multivariate geostatistical modelling of the series in time, the MAFs method allows a quite direct treatment. The paper showed the utility of MAFs for combining, selecting or forecasting indicator time series to assist in the assessment of fish stocks using a suite of survey-based population indicators. The procedures were applied to indicator time series of North Sea cod.

The results were that the immature part of the stock showed simultaneous change in its spatial organisation (MAF 1) and abundance since 2000. The MAF selected indicators that showed a continuous trend with no signal of an inversion. L50 at maturity continuously declined since 1985 . Latitude of the centre of gravity of mature cod continuously shifted northwards from 1988. Abundance of mature fish declined in two steps: rapidly in the late 80 s and then in the period following 2000. The spatial organisation of immature and mature cod changed rapidly until the mid-90s and then fluctuated. The results were consistent with assessments carried out by ICES (e.g. 2003), which showed that the stock has been below the abundance reference point since 1999. The present analysis broadened the biological basis of the assessment. A northerly shift in the centre of gravity of North Sea cod spatial distribution had already been reported (Rindorf and Lewy 2006; Hedger et al. 2004). The present analysis was consistent with this finding; but also revealed that more changes occurred in the spatial distribution (microstructure, anisotropy and occupation area) in addition to the change in centre of gravity. The short-term forecast for North Sea cod was a continuation of the historical trend. The short-term forecast is probably sensitive to the last MAF values of the times series. The same procedure applied to the anchovy in the Bay of Biscay (Woillez 2007; Woillez et al. 2007b) forecasted a change from the past years.

Acknowledgements. The study was carried out with the financial support of the European Union (project FISBOAT, 2004-2007, Fisheries Independent Survey-Based Operational Assessment Tools, Contract 502572).

\section{Supporting information}

Appendix S1. R-script functions MAF_functions.r Appendix S2. R-script MAF_combine.r.
This script needs codNS_85-04_Groups\&Spa.txt data file. Appendix S3. R-script MAF_select.r.

This script works with codNS_85-04_Groups\&Bio\&Spa.txt data file.

Appendix S4. R-script MAF_forecast.r.

The script runs with the data file codNS_85-05_Groups\&Bio\&Spa.txt.

Appendix S5. Table codNS_85-04_Groups\&Spa.txt

Appendix S6. Table codNS_85-04_Groups\&Bio\&Spa.tx

Appendix S7. Table codNS_85-05_Groups\&Bio\&Spa.txt

Supporting information is only available in electronic form at www.alr-journal.org

\section{References}

Bouleau M., 2005, Combinaison géostatistique de l'acoustique et des captures dans les campagnes scientifiques de pêche par chalutage. Thèse dr Géostatistique, Ecole Nationale Supérieure des Mines, Paris.

Conradsen K., Ersboll B.K., Thyrsted T., 1985, A comparison of $\mathrm{min} / \mathrm{max}$ autocorrelation factor analysis and ordinary factor analysis. Nordic Symposium in Applied Statistics, Lyngby, pp. 47-56.

Cotter J., Mesnil B., Witthames P., Uriarte A., Parker-Humphreys M., 2009, Notes on nine biological indicators estimable from trawl surveys with an illustrative assessment for North Sea cod. Aquat. Living Resour. 22, 135-153.

Desbarats A.J., 2001, Geostatistical modelling of regionalized grainsize distributions using $\mathrm{min} / \max$ autocorrelation factors. In: Monestiez P., Allard D., Froidevaux R. (Eds.) Geostatistics for Environmental Applications III, Kluwer Academic Publisher, pp. 441-452.

Desbarats A.J., Dimitrakopoulos R., 2000, Geostatistical simulation of regionalized pore-size distributions using $\mathrm{min} / \mathrm{max}$ autocorrelation factors. Math. Geol. 32, 919-942.

Erzini K., 2005, Trends in NE Atlantic landings (southern Portugal): identifying the relative importance of fisheries and environmental variables. Fish. Oceanogr. 14, 195-209.

Erzini K., Inejih C.A.O., Stobberup K.A., 2005, An application of two techniques for the analysis of short, multivariate non-stationary time-series of Mauritanian trawl survey data. ICES J. Mar. Sci. 62, 353-359.

Hedger, R., McKenzie, E., Heath, M., Wright, P., Scott, B., Gallego, A., Andrews, J. 2004, Analysis of the spatial distribution of mature cod (Gadus morhua) and haddock (Melanogrammus aeglefinus) abundance in the North Sea (1980-1999) using generalised additive models. Fish. Res. 70, 17-25.

ICES, 2003, Report of the ICES Advisory Committee on Fishery Management, ICES Coop. Res. Rep. 261.

Jennings S., 2005, Indicators to support an ecosystem approach to fisheries. Fish Fish. 6, 212-232.

Löfgren K.-G., Ranneby B., Sjöstedt S., 1993, Forecasting the business cycle without using minimum autocorrelation factors. J. Forecasting 12, 481-498.

Pearce K.F., Frid C.L.J., 1999, Coincident changes in four components of the North Sea ecosystem. J. Mar. Biol. Assoc. UK 79, 183-185.

$\mathrm{R}$ development Core Team, 2005, R: A language and environment for statistical computing. Vienna, Austria, R Foundation for Statistical Computing. URL http://cran.r-project.org/

Rindorf, A., Lewy, P., 2006, Warm windy winters drive cod north and homing keeps them there. J. Appl. Ecol. 43, 445-453. 
Shapiro D.E., Switzer P., 1989, Extracting time trends from multiple monitoring sites. Department of Statistics, Stanford University. Tech. Rep. 132.

Solow A.R., 1994, Detecting change in the composition of a multispecies community. Biometrics 50, 556-565.

Switzer P., Green A.A., 1984, Min/max autocorrelation factors for multivariate spatial imaging. Department of Statistics, Stanford University, Tech. Rep. 6.

Woillez M., 2007, Contributions géostatistiques à la biologie halieutique. Thèse dr. Géostatistique, Ecole Nationale Supérieure des Mines, Paris.

Woillez M., Poulard J-C., Rivoirard J., Petitgas P., Bez N., 2007a, Indices for capturing spatial patterns and their evolution in time, with application to European hake (Merluccius merluccius) in the Bay of Biscay. ICES J. Mar. Sci. 64, 537-550.
Woillez M., Rivoirard J., Petitgas P., 2007b, Selecting and combining survey-based indices of fish stocks using their correlation in time to make diagnostics of their status. ICES CM 2007/O:07.

Woillez M., Rivoirard J., Petitgas P., 2009, Notes on survey-based spatial indicators for monitoring fish populations. Aquat. Living Resour. 22, 155-164.

Zuur A.F., Pierce G.J., 2004, Common trends in Northeast Atlantic squid time series. J. Sea Res. 52, 57-72.

Zuur A.F., Fryer R.J., Jolliffe I.T., Dekker R., Beukema J.J., 2003a, Estimating common trends in multivariate time series using dynamic factor analysis. Environmetrics 14, 665-685.

Zuur A.F., Tuck I.D., Bailey N., 2003b, Dynamic factor analysis to estimate common trends in fisheries time series. Can. J. Fish. Aquat. Sci. 60, 542-552. 